\title{
Study of Rayleigh-Backscattering Induced Coherence Collapse in an Asymmetric DFB FL Sensor
}

\author{
Wen $\mathrm{LIU}^{1 *}$, Lina $\mathrm{MA}^{2}$, Zhengliang $\mathrm{HU}^{2}$, Ying $\mathrm{FENG}^{1}$, and Huayong YANG ${ }^{1}$ \\ ${ }^{1}$ College of Optoelectronic Science and Engineering, National University of Defense Technology, Changsha, 410073, \\ China \\ ${ }^{2}$ Academy of Ocean Science and Engineering, National University of Defense Technology, Changsha, 410073, China \\ *Corresponding author: Wen LIUＥ-mail: liusenanbei@163.com
}

\begin{abstract}
Rayleigh-back scattering induced coherence collapse of an asymmetric distributed feedback fiber laser (DFB FL) sensor is investigated using a composite cavity model. The coherence collapse threshold condition of the asymmetric DFB FL sensor is measured. The DFB FL sensor shows different dynamic behaviors in different pump configurations. According to the asymmetric behavior to the external optical feedback, a novel method to find the actual phase shift position of the asymmetric DFB FL sensor is presented.
\end{abstract}

Keywords: Asymmetric DFB fiber laser sensor; phase shift; coherence collapse; Rayleigh backscattering

Citation: Wen LIU, Lina MA, Zhengliang HU, Ying FENG, and Huayong YANG, "Study of Rayleigh-Backscattering Induced Coherence Collapse in an Asymmetric DFB FL Sensor," Photonic Sensors, 2016, 6(3): 209-213.

\section{Introduction}

Distributed feedback fiber lasers (DFB FLs) have attracted general attention since 1990s, because of the impact structure, low noise figure, and the inherent wavelength-encoded multiplexing capability [1-4]. The laser frequency of a DFB FL is determined by the resonance condition of the laser cavity, which is sensitive to the physical parameters that could disturb the oscillation process. By using the dependence of the laser frequency on the physical parameters, DFB FL can be employed as a sensor [5]. DFB FL sensor arrays are normally arranged through remote pumping and interrogation configuration, which means that a long lead fiber is required to feed the pump light into DFB FLs [6]. In the lead fiber, Rayleigh backscattering may cause excess frequency noise or even unstable output of the DFB FLs, namely, coherence collapse [7], and substantially affects the performance of DFB FL sensors. The maximum multiplexing capacity of DFB FL array remains to be 16 until now [8], and coherence collapse induced by Rayleigh backscattering is considered as one of the main reasons for the limited array scale [9]. To improve the performance of the DFB FL sensor array, it is necessary to study the effect of coherence collapse induced by Rayleigh scattering of the lead fiber on the operation status of the DFB FL. The tolerable length of lead fiber was found to be $135 \mathrm{~m}-200 \mathrm{~m}$ for symmetric DFB FL [6], corresponding to a typical Rayleigh backscattering of $-72 \mathrm{~dB} / \mathrm{m}$, while the dynamic behavior of asymmetric DFB FL with external optical feedback was rarely mentioned.

In this paper, we study the tolerance of asymmetric DFB FL to Rayleigh backscattering of a 
long lead fiber. We start to analyze the coherence collapse threshold condition of asymmetric DFB FL in Section 2. The dynamic behaviors of an asymmetric DFB FL sensor to Rayleigh backscattering is evaluated in different pump configurations in Section 3. In Section 4, a novel method to determine the phase shift position of asymmetric DFB FL is proposed based on the coherence-collapse threshold length measurement.

\section{Composite cavity model}

DFB FL consists of a phase-shift Bragg grating in a short piece of rare earth doped fiber. The symmetric grating structure is designed for DFB FL to obtain the single polarization laser output and reduce the pump threshold [10], but many asymmetric DFB FLs are made on purpose. In a sensor array, DFB FLs with asymmetrical outputs can modify the pump power distribution and improve the multiplexing capacity with fixed pump power budget $[11,12]$.

In asymmetric DFB FL, each grating segment on either side of the phase shift can be considered as a separate reflector. By simplifying the distributed feedback cavity to be a Fabry-Perot (FP) structure and considering the external optical feedback as the external cavity of the FL [13], a composite cavity model can be established as shown in Fig. 1. $r_{1}$ and $r_{2}$ are the reflection coefficients of the FP cavity, $r_{\mathrm{ext}}$ is the reflection coefficient of the external reflection facet, $L$ is the effective length of the FP cavity, and $d$ is the external path length.

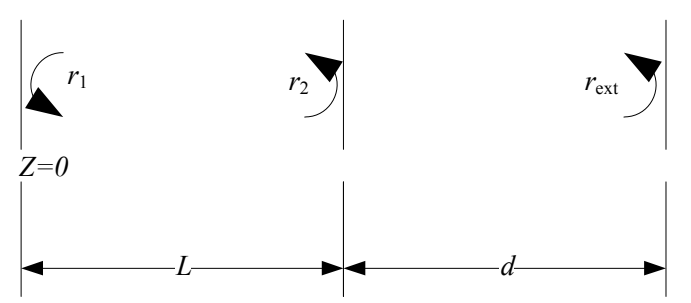

Fig. 1 Schematic diagram of the composite cavity model.

The composite cavity can be equivalent to an FP cavity, and the reflection coefficients of the equivalent FP cavity can be written as

$$
\left\{\begin{array}{l}
r_{L}=r_{1} \\
r_{R}=r_{2}\left[1+\frac{T_{2} r_{\mathrm{ext}} \cos \left(2 \pi \nu \tau_{e}\right)}{r_{2}}\right]-\mathrm{i} T_{2} r_{\mathrm{ext}} \sin \left(2 \pi \nu \tau_{e}\right)
\end{array}\right.
$$

where $T_{2}$ is the transmissivity of right piece of uniform grating segment, and $\tau_{\mathrm{e}}$ is the external round trip delay.

The steady-state laser oscillation condition for the equivalent FP cavity can be given as

$$
\begin{gathered}
\left|r_{L}\right|\left|r_{R}\right| \exp \left[\left(g_{c}-\gamma\right) L\right]=1 \\
\Delta \varphi=0=\frac{\tau_{L}}{\tau_{e}}\left[2 \pi \tau_{e}\left(v-v_{0}\right)+\right. \\
\left.\frac{T}{r_{2}} \frac{\tau_{e}}{\tau_{L}} \sqrt{1+\alpha^{2}} r_{\text {ext }} \sin \left(2 \pi v_{0} \tau_{e}+2 \pi \Delta v \tau_{e}-\theta_{\alpha}\right)\right]
\end{gathered}
$$

where $v_{0}$ is the laser frequency without optical feedback, $\tau_{L}$ is the roundtrip time of the solitary laser, $\alpha$ is the linewidth enhancement factor, and $\theta_{\alpha}$ is the associated phase. The frequency shift $\Delta v$ due to the optical feedback and the feedback parameter $C$ can be expressed as

$$
\begin{gathered}
\tau_{e} \Delta \omega=-C \sin \left(\Delta \omega \tau_{e}+2 \pi v_{0} \tau_{e}-\theta_{\alpha}\right) \\
C=\frac{T}{r_{2}} \frac{\tau_{e}}{\tau_{L}} \sqrt{1+\alpha^{2}} r_{\text {ext }} .
\end{gathered}
$$

For very weak feedback with $C<1$, (4) has only one solution. When $C \geq 1$, there are more than one solutions for (4), indicating that the external optical feedback splits the single mode operation into multiple external cavity modes, accompanying with dramatically increased noise level and intensity fluctuation, resulting in coherence collapse. As a result, $C=1$ may be defined as the coherence collapse threshold condition for DFB FL.

For an asymmetric DFB FL, the reflection coefficient and the transmissivity of each grating segment at the Bragg wavelength are given by [13]

$$
\begin{gathered}
r_{i} \approx-\tanh \left(-\kappa l_{i}\right) \\
T_{i} \approx 4 \exp \left(-\kappa l_{i}\right)
\end{gathered}
$$

where $l_{\mathrm{i}}$ with $i=1,2$ is the length of the grating segment. The effective cavity length of DFB FL can be written as

$$
L \approx\left(r_{1}+r_{2}\right) / 2 \kappa
$$


By substituting (7) and (8) into (5), the feedback parameter of asymmetric DFB FL can be rewritten as

$$
C=\frac{8 \kappa \sqrt{1+a^{2}} r_{\text {ext }} d \exp \left(-2 \kappa l_{2}\right)}{r_{2}\left(r_{1}+r_{2}\right)} .
$$

For a DFB FL sensor system, the external optical feedback mainly comes from external reflecting facet, splice points, and Rayleigh backscattering [14]. The influence of former two sources can be eliminated to the negligible extent, but the detrimental effect of the last one is never possible to be entirely ignored, which becomes the main factor to limit the performance of the DFB FL sensor. Then the coherence collapse threshold length of lead fiber in the DFB FL system can be given by

$$
d=\left(\frac{r_{2}\left(r_{1}+r_{2}\right)}{8 \kappa \sqrt{1+a^{2}} r_{R} \exp \left(-2 \kappa l_{2}\right)}\right)^{\frac{2}{3}} .
$$

When $l_{1}=l_{2}$, (10) can be used to calculate the threshold condition for symmetric DFB FL, which coincides with the conclusion got by E. Rønnekleiv et al. [6].

\section{Experiments and results}

A high resolution frequency shift demodulation system is constructed to evaluate the influence of external optical feedback on the asymmetric DFB FL sensor. The schematic diagram is shown in Fig. 2. The DFB FL sensor is a fiber laser hydrophone with ultrahigh pressure sensitivity [15]. The total length of the laser cavity is $40 \mathrm{~mm}$, and the phase shift section is $4 \mathrm{~mm}$ long. The DFB FL sensor is pumped by a $975-\mathrm{nm}$ semiconductor laser at $63 \mathrm{~mW}$ through a 980/1550 WDM (wavelength division multiplexer). The output signal of the FL sensor is guided to an unbalanced Michelson interferometer through an isolator. The optical path difference of the interferometer is $15 \mathrm{~m}$. A sinusoidal signal at the frequency of $12.5 \mathrm{kHz}$ is loaded to the piezoelectric ceramic transducer (PZT) in one arm of the interferometer. The interference signal is converted into electric voltage and then acquired by an acquisition card. A phase generated carrier (PGC) scheme is adopted to achieve high resolution interrogation. The whole optical setup is packaged to isolate the environment fluctuation, and the end facet of the pigtail is immersed in the refractive index matched gel to eliminate the back reflection.

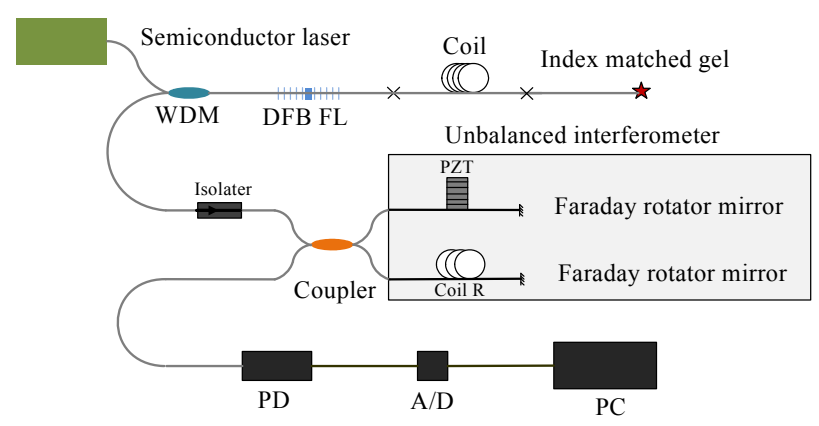

Fig. 2 Schematic diagram of the frequency shift demodulation system.

The distance between the WDM and DFB FL sensor is set to $1 \mathrm{~m}$, while the lead fiber on the other end of the DFB FL sensor is increased by $10 \mathrm{~m}$ each time until coherence collapse occurs. The two ends of the asymmetric DFB FL sensor are labeled as Port 1 and Port 2, and the DFB FL sensor is pumped through Port 1 at first. The phase noise floor of the DFB FL sensor with different lengths of lead fiber is shown in Fig. 3, and the demodulated interference signals are shown in Fig. 4.

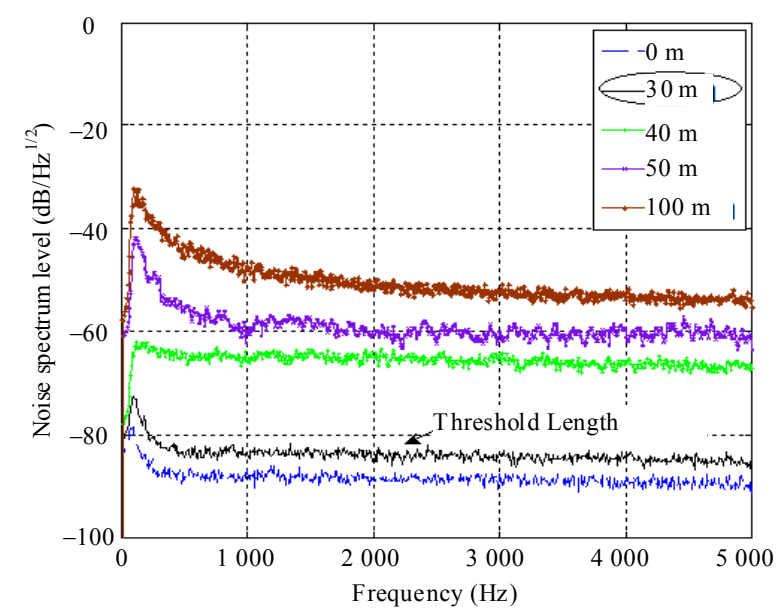

Fig. 3 Phase noise level of DFB FL sensor due to an increase in the length of lead fiber.

It is illustrated that the phase noise level of the DFB FL sensor rises with an increase in the length of the lead fiber. When no extra lead fiber is added 
to the pigtail, the phase noise of the DFB FL sensor is about $-89 \mathrm{~dB}$ re. rad/ $/ \mathrm{Hz}^{1 / 2} @ 1 \mathrm{kHz}$. When the lead fiber is $30 \mathrm{~m}$ long, the phase noise increases by $5 \mathrm{~dB}$, and the interference signal is still stable as shown in Fig. 4. However, when another $10-\mathrm{m}$ lead fiber increases, the phase noise increases by over $10 \mathrm{~dB}$, and there is self-pulsing in the interference signal, hinting that coherence collapse occurs.
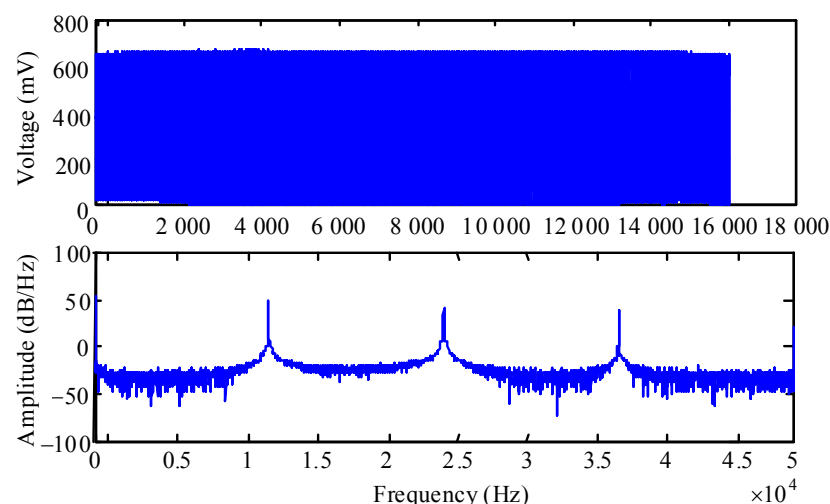

(a)
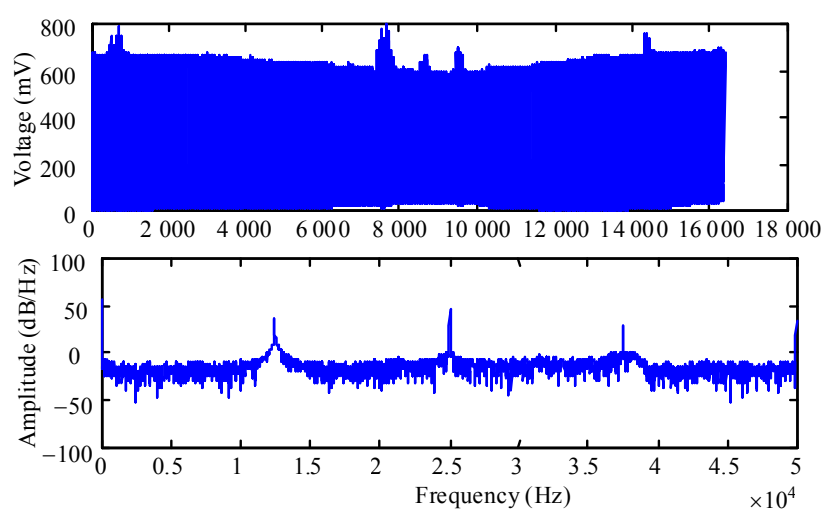

(b)

Fig. 4 Demodulated interference signal with different length of lead fiber: (a) $d=30 \mathrm{~m}$ and (b) $d=40 \mathrm{~m}$.

Through a similar approach, the coherence collapse threshold length of the lead fiber of the DFB FL sensor is measured when pumped from Port 2. The threshold length and the laser output power of the DFB FL sensor in different pump configurations are presented in Table 1.

Table 1 Coherence collapse threshold length and laser output power of the asymmetric DFB FL sensor.

\begin{tabular}{ccc}
\hline Pump port & Port 1 & Port 2 \\
\hline Threshold length $(\mathrm{m})$ & 14.13 & 43.89 \\
Output power $(\mu \mathrm{W})$ & 30 & 100 \\
\hline
\end{tabular}

It is indicated that the asymmetric DFB FL sensor has distinctive threshold lengths of the lead fiber in different pump configurations, and the difference of the threshold length exceeds $50 \mathrm{~m}$. When the DFB FL sensor system is arranged through a remote-pumping and interrogation configuration, the DFB FL sensor should be pumped from Port 2 to extend the total length of the sensing system.

\section{Phase shift assessment method based on coherence collapse threshold length}

Based on (6) and (10), the coherence collapse threshold length ratio of the asymmetric DFB FL in different pump configurations satisfies

$$
\frac{d_{1}}{d_{2}}=\left[\frac{\tanh \left(\kappa l_{2}\right)}{\tanh \left(\kappa l_{1}\right)} \exp \left(2 \kappa l_{1}-2 \kappa l_{2}\right)\right]^{2 / 3} .
$$

For an asymmetric DFB FL with 40-mm cavity length and 4-mm phase shift section, the threshold length ratio to the grating segment length near Port 1 is shown in Fig. 5, where the estimated grating coupling coefficient is $\kappa=150 \mathrm{~m}^{-1}$.

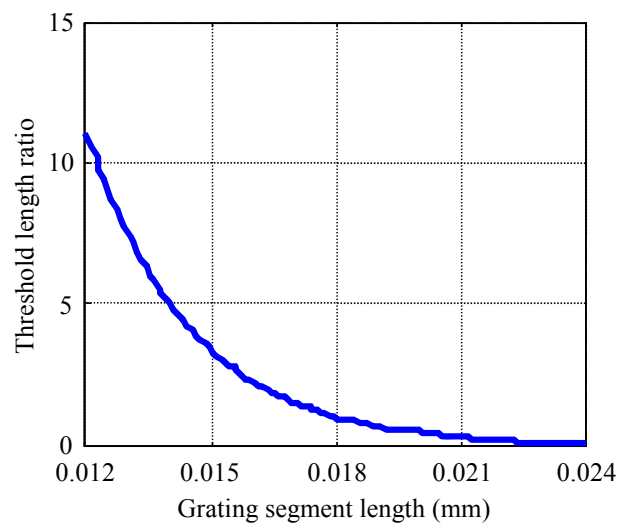

Fig. 5 Phase noise level of the DFB FL sensor due to an increase in the external path length.

As the threshold length ration of the DFB FL sensor is $10 / 3$, the lengths of grating segments beside of the phase shift section can be deduced as $15 \mathrm{~mm}$ and $21 \mathrm{~mm}$, respectively.

In the field test, the performance of the DFB FL sensor always differs from the initial DFB FL before mounting, as non-uniform extension or twisting of the laser cavity is introduced by the mounting 
structure. The method proposed here can be used to understand the real physical state of the FL cavity, and help to optimize the sensor performance.

\section{Conclusions}

The stability of the asymmetric DFB FL sensor with Rayleigh scattering of the lead fiber is studied. The coherence collapse threshold length of an asymmetric DFB FL sensor is measured based on a high resolution frequency shift demodulation system. The difference in the threshold length of the same FL sensor in different pump configurations suggests a novel method to attain the phase shift position, which is significant to enlarge the total length of the DFB FL sensor system. More details need to be revealed for the asymmetric DFB FL with different phase shifts at different positions, and the coherence collapse characteristics of the asymmetric DFB FL sensor array will be studied in further work.

\section{Acknowledgment}

This work was supported by the National Science Foundation of China (NSFC 11304388).

Open Access This article is distributed under the terms of the Creative Commons Attribution 4.0 International License (http://creativecommons.org/ licenses/by/4.0/), which permits unrestricted use, distribution, and reproduction in any medium, provided you give appropriate credit to the original author(s) and the source, provide a link to the Creative Commons license, and indicate if changes were made.

\section{References}

[1] J. T. Kringlebotn, J. L. Archambault, L. Reekie, and D. N. Payne, " $\mathrm{Er}^{3+}: \mathrm{Yb}^{3+}$-codoped fiber distributedfeedback laser," Optics Letters, 1994, 19(24): 2101-2103.

[2] D. J. Hill, P. J. Nash, D. A. Jackson, D. J. Webb, S. F. O'Neill, I. Bennion, et al., "A fiber laser hydrophone array," Proc. SPIE, 1999, 3860: 55-66.

[3] D. J. Hill, J. D. Hodder, S. D. Freitas, S. D. Thomas, and L. Hickey, "DFB fibre laser sensor developments," Proc. SPIE, 2005, 5855: 904-907.

[4] G. A. Cranch, G. M. H. Flockhart, and C. K. Kirkendall, "Distributed feedback fiber laser strain sensors," IEEE Sensors Journal, 2008, 8(7): 1161-1172.

[5] Y. Liu, W. Zhang, T. Xu, J. He, F. Zhang, and F. Li, "Fiber laser sensing system and its applications," Photonic Sensors, 2011, 1(1): 43-53.

[6] E. Rønnekleiv, O. Hadeler, and G. Vienne, "Stability of an Er-Yb-doped fiber distributed-feedback laser with external reflections," Optics Letters, 1999, 9(24): 617-619.

[7] R. W. Tkach, "Regimes of feedback effects in $1.5 \mu \mathrm{m}$ distributed feedback lasers," Journal of Lightwave Technology, 1986, 4(11): 1655-1661.

[8] S. Foster, A. Tikhomirov, M. Englund, H. Inglis, G. Edvell, and M. Milnes, "A 16 channel fibre laser sensor array," in $A C O F T / A O S, 2006$ : 40-42.

[9] A. Tikhomirov and S. Foster, "DFB FL sensor multiplexing noise," in ACOFT/AOS, 2006: 60-62.

[10] M. Yamada and K. Sakuda, "Analysis of almost-periodic distributed feedback slab waveguides via a fundamental matrix approach," Applied Optics, 1987, 26(16): 3474-3478.

[11] P. Wang, J. Chang, C. Zu, B. Sun, G. Lv, S. Zhang, et al., "A four-element sensor array consisting of asymmetric distributed-feedback fiber lasers," Photonic Sensors, 2014, 4(2): 180-187.

[12] H. F. Qi, Z. Q. Song, S. J. Li, J. Guo, C. Wang, and G. D. Peng, "Apodized distributed feedback fiber laser with asymmetrical outputs for multiplexed sensing applications," Optics Express, 2013, 21(9): 11309-11314.

[13] F. Favre, "Theoretical analysis of external optical feedback on DFB semiconductor lasers," IEEE Journal of Quantum Electronics, 1995, 1(23): 81-88.

[14] K. Yelen, L. M. B. Hickey, and M. N. Zervas, “A new design approach for fiber DFB lasers with improved efficiency," IEEE Journal of Quantum Electronics, 2011, 40(6): 711-720.

[15] L. Ma, Y. M. Hu, H. Luo, and Z. L. Hu, "DFB fiber laser hydrophone with flat frequency response and enhanced acoustic pressure sensitivity," IEEE Photonics Technology Letters, 2009, 21(17): 1280-1282. 